\title{
Phenolic profile and antioxidant activity of olive fruits of the Turkish variety "Sarıulak" from different locations
}

\author{
By D. Arslan ${ }^{\star}$ and M.M.Özcan \\ Selçuk University, Faculty of Agriculture, Department of Food Engineering, 42030, Konya, Turkey \\ ("corresponding author: dears@ selcuk.edu.tr)
}

\begin{abstract}
RESUMEN
Perfil fenólico y actividad antioxidante de aceitunas de la variedad turca "Sarıulak" de diferentes procedencias.

Se han estudiado los compuestos fenólicos, el efecto captador de radicales y la capacidad antioxidante de aceitunas de la variedad "Sarıulak" procedentes de cuatro localidades diferentes, Alanya, Ceyhan, Silifke y Karaman de la región mediterránea de Turquía, que fueron recogidas en tres diferentes fechas de cosecha (HDs) y en dos años consecutivos de cultivos. Los niveles de la mayoría de estos compuestos fenólicos, expresados en $\mathrm{mg} / \mathrm{kg}$, de los frutos procedentes de Alanya fueron los mas altos entre los valores obtenidos de todos los lugares, principalmente en primer HDs, por ejemplo, el hidroxitirosol llega a 3596,4 , la luteolina a 269,5 , el ácido vanílico a 159,8 y el ácido cafeico a 62,1. Las aceitunas de Alanya, que tenían el mayor promedio de lluvias de las otras localidades, presentó el mayor contenido de fenoles. Las aceitunas de Silifke, que tuvo una dramática disminución de las precipitaciones entre dos años de cultivo, mostraron una reducción en el contenido de fenoles totales en la campaña agrícola siguiente. Los contenidos más alto de oleuropeína fueron detectados en las muestras de frutos cultivadas en Silifke $(963,5$ a $2.981,8 \mathrm{mg} / \mathrm{kg})$ y Karaman $(835.2-655.6 \mathrm{mg} / \mathrm{kg})$. Todas las localidades mostraron cambios similares en la actividad antioxidante que apunta a un aumento con HDs. El efecto de HD en los compuestos fenólicos como el 4-hidroxifenil-acético, ácido cafeico, ácido siríngico, hidroxitirosol, luteolina, ácido clorogénico y el ácido 3,4-dihidroxi fueron más dependientes del factor localización.
\end{abstract}

PALABRAS CLAVE: Aceitunas - Antioxidante - Cosecha-Fenoles - Localización - Variedad.

\section{SUMMARY}

Phenolic profile and antioxidant activity of olive fruits of the Turkish variety "Sarıulak" from different locations.

The phenolic compounds, radical scavenging effect and antioxidant capacity of olive fruits from the "Sarıulak" variety were studied from four different locations: Alanya, Ceyhan, Silifke and Karaman in the Mediterranean region of Turkey. They were collected on three different harvest dates (HDs) and in two consecutive crop years. The levels of most of the phenolic compounds in the fruits of the Alanya location were remarkably high among the values $(\mathrm{mg} / \mathrm{kg}$ ) obtained for all location samples mainly on the first HDs, for instance hydroxytyrosol rose to 3596.4 , luteolin rose to 269.5 , vanillic acid rose to 159.8 and caffeic acid rose to 62.1. The olive fruits from Alanya, which had the highest average rainfall compared to the other locations, showed the highest phenolic content.
The olive fruits from Silifke, which had a dramatic decrease in rainfall between the two crop years, showed a reduction in total phenolic content in the following crop year. The highest oleuropein contents $(\mathrm{mg} / \mathrm{kg})$ were detected for the fruit samples grown in Silifke (963.5-2981.8) and for Karaman (835.2-655.6). All of the locations showed similar changes in antioxidant activity pointing to an increase with later HDs. The effect of HD on the phenolic compounds such as 4-hydroxyphenyl acetic acid, caffeic acid, syringic acid and hidroxytyrosol, luteolin, chlorogenic acid and 3,4-dihydroxybenzoic acid were more dependent on the location factor.

KEY-WORDS: Antioxidant - Harvest - Location - Olive fruit - Phenolics - Variety.

\section{INTRODUCTION}

Olea europaea is one of the most important and widespread fruit trees in the Mediterranean basin which is cultivated in southern European countries bordering the Mediterranean Sea and in North Africa (Loumou and Giourga, 2003). Although Turkey is the fourth country in the production of fresh olives coming after Spain, Italy and Greece, it is the second greatest producer in the world for table olives after Spain (Tetik, 1989; Ünal and Nergiz, 2003). According to 2004 statistics, there are around 107 million olive trees in Turkey, of which $32 \%$ are used for edible olive production and $68 \%$ are used for olive oil production (ZAE, 2007; Öğütçü et al., 2008). There are five distinct olive growing regions: the Aegean region (55.11\%), the Marmara Region (27.72\%), the Mediterranean Region (14.94\%) and the Black Sea Region (2.22\%). Around $75-80 \%$ of the total olive oil production in Turkey is located within the Aegean region (Gurbuz et al., 2004; DiE, 2008).

The Sarıulak variety comprises $6 \%$ of the olive tree number cultivated in the Mediterranean region of Turkey and covers $0.6 \%$ of the total olive tree number in Turkey. The geographical origin of this variety is Tarsus and it has spread to the İcel, Erdemli, Gülnar, Seyhan, Kazan and Yumurtalık locations of the Mediterranean region of Turkey. It is characterized by high vigor, low frost resistance, medium-sized fruit and medium productivity. It is generally used in producing green or black table olives (Anonymous, 1991; Özkaya, 2003). 
The olive fruit is rich in phenolic compounds and there has been a growing interest in this group due to their antioxidant activity and health benefits (Ryan et al., 2002). The most important classes of phenolic compounds in olive fruits include phenolic acids, phenolic alcohols, flavonoids and secoiridoids (SolerRivas et al., 2000). The phenolic alcohols of olives are 3,4-dihydroxyphenylethanol (hydroxytyrosol or h-tyrosol) and p-hydroxyphenylethanol (tyrosol) (Ryan and Robards, 1998; Romero et al., 2002); flavonoids include luteolin 7-O-glucoside, rutin and apigenin 7-O-glucoside, and the anthocyanins, cyanidin 3-O-glucoside and cyanidin 3-O-rutinoside (Esti et al., 1998; Romani et al., 1999); secoiridoids such as oleuropein and ligstroside; some oleuropein derivatives, namely demethyloleuropein, oleuropein aglycone and elenolic acid (Servili et al., 2004) and the main hydroxycinnamic acid derivative, verbascoside (Romani et al., 1999). There have been few works published on the characteristics of Turkish monovarietal olive fruits, and none of them reports on the concentrations of phenolic compounds (Nergiz and Engez, 2000; Tanilgan et al., 2007; Ocakoğlu et al., 2009). There is only one published on Sarıulak which reports about the antioxidant activity of this variety (Keçeli and Gordon, 2001).

The aim of this work was to determine the phenolic profile and antioxidant activity of the fruits of the Turkish olive variety "Sarıulak" from four different locations of South Anatolia and to improve local and international knowledge on this variety. The effects of harvest date and harvest year on these parameters were also discussed.

\section{MATERIALS AND METHODS}

\subsection{Olive origin}

The present work was carried out on monovarietal olive fruits from the Turkish variety; Sarıulak. The olive samples were collected from different olive groves located in the olive growing countryside areas of the southern region of Turkey (the Mediterranean Region and the Southeast Anatolia). The locations where the olive samples were obtained were Alanya (in the city of Antalya), Silifke (in the city of Mersin), Bucakkışla (in the city of Karaman) and Ceyhan (in the city of Adana). The general climate of these areas is moderate, common to the Mediterranean region. The characteristics of the production areas of the olive samples studied are reported in Figure 1. Climatic data (temperature and rainfall) were obtained for the two experimental years (2006-2007) from the Turkish State Meteorological Service (DIE, 2008). The altitude values of the locations are also given.

The olive fruits were hand picked on three different HDs, corresponding to between the 15th of September and the 1st of October (1st HD), the 20th of October and the 1st of November (2nd HD) and the 20th of November to the 10th of December (3rd $\mathrm{HD}) .5 \mathrm{~kg}$ of olives were collected from the same trees for each HD. Only healthy fruits without any kind of infection or physical damage were selected. After harvesting, the olive fruits were immediately transported to the laboratory in cool bags.

The olive ripening index (RI) was determined according to the method developed by Boskou (1996) based on the evaluation of the olive skin and pulp

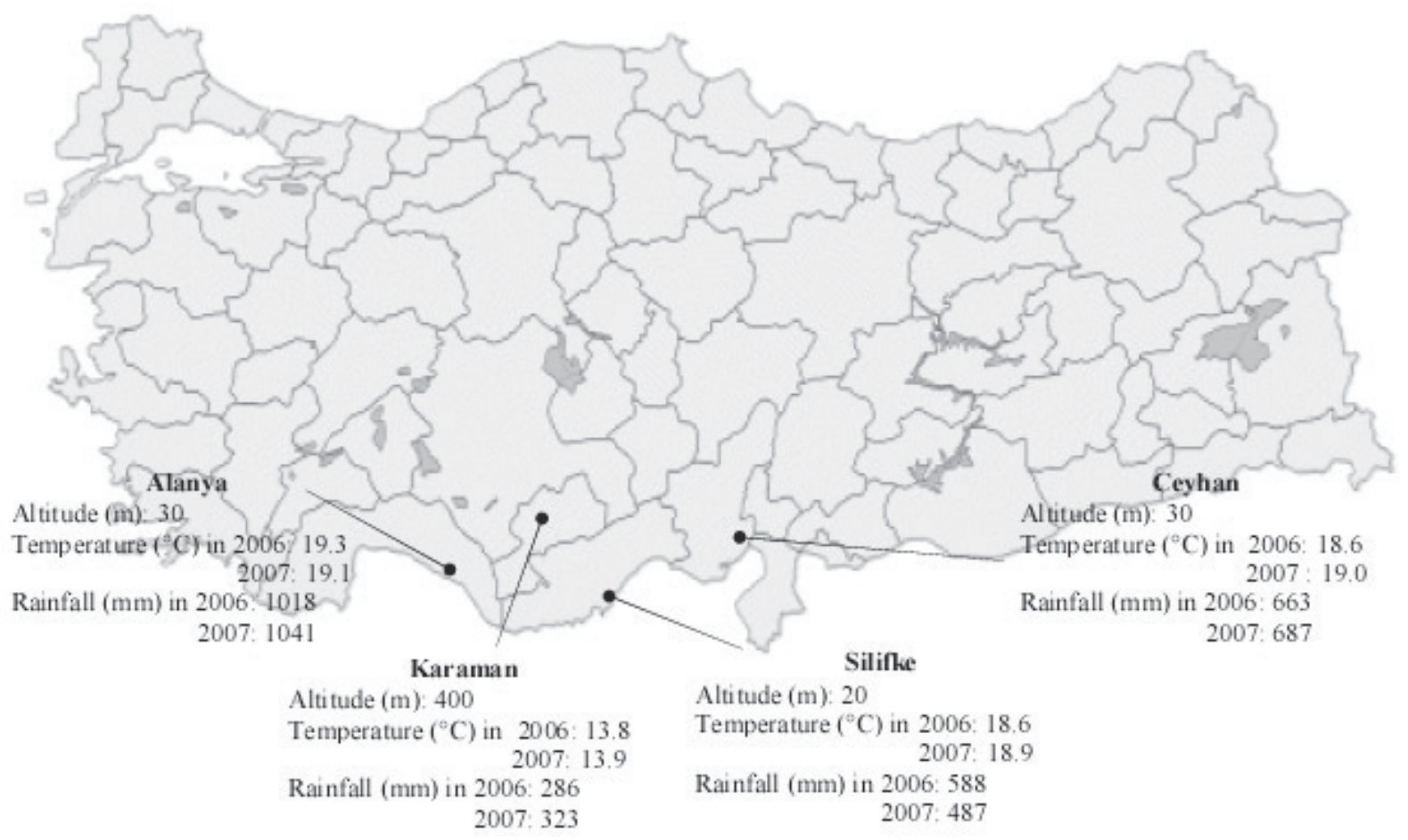

Figure 1

Climatic characteristics and altitudes of the locations where the olive fruits were obtained 
colors. RI values range from $0(100 \%$ intense green skin) to 7 (100\% purple flesh and black skin). The ripening indexes of the olives are given in Table 1.

\subsection{Extraction of phenolic compounds}

The phenolic extracts of the olive fruits were obtained using the method of Vinha et al., (2005) with modifications. The extraction, purification and separation were done as follows: $1.5 \mathrm{~g}$ of sample were extracted with $20 \mathrm{ml}$ of $80: 20(\mathrm{v} / \mathrm{v})$ methanol-water. The mixture was homogenized using an Ultraturrax homogenizer (IKA, Germany), centrifuged at 3000 $\mathrm{rev} / \mathrm{min}$. for $5 \mathrm{~min}$ and the supernatant was filtered through filter paper. The pellet was re-extracted as above. The purification was carried out with $10 \mathrm{~mL}$ of $n$-hexane added to eliminate the residual oil of the resulting methanolic extract. The separation of phases was performed using separating funnels. The methanolic extracts were combined (phenolic extract) and injected for HPLC analysis.

\subsection{HPLC analysis of phenolic compounds}

The extracted phenolic fractions were analyzed by HPLC. The HPLC system included an LC 10A vp, an LC-20AT prominence pump, a CTO-10AS VP heater (column temperature $22^{\circ} \mathrm{C}$ ), a SIL$20 \mathrm{~A}$ prominence autosampler and an SPD-M20A Prominence diode-array detector (Shimadzu, Kyoto, Japan). The column was an Inertsil ODS$3(5 \mu \mathrm{m}, 25 \mathrm{~cm} \times 4.6 \mathrm{~mm}$ i.d.) (GL Sciences, Tokyo, Japan). PC running Class VP chromatography manager software (Shimadzu, Japan) was used and chromatograms were obtained at 280 and $320 \mathrm{~nm}$. The eluents were $2 \%$ aqueous formic

Table 1

Ripening index* of olive fruit

\begin{tabular}{cccc}
\hline Location & H.D. $^{\dagger}$ & $\mathbf{2 0 0 6}$ & $\mathbf{2 0 0 7}$ \\
\hline \multirow{3}{*}{ Alanya } & 1 & 0.76 & 1.13 \\
& 2 & 2.87 & 2.74 \\
& 3 & 5.69 & 5.97 \\
Karaman & 1 & 1.05 & 1.08 \\
& 2 & 3.44 & 3.23 \\
Ceyhan & 3 & 5.89 & 4.88 \\
& 1 & 1.40 & 1.21 \\
& 3 & 3.71 & 2.78 \\
Silifke & 1 & 5.82 & 5.75 \\
& 2 & 1.20 & 1.06 \\
& 3 & 6.49 & 3.39 \\
\hline
\end{tabular}

* Ripening index of olive fruits ranging from 0 to 7.0 represents $100 \%$ intense green skin and 7 represents $100 \%$ purple flesh and black skin.

${ }^{\dagger}$ Harvest date: 1: 15 th of September-1st of October (1st HD); 2: 20th October -1st of November (2nd HD); 3: 20th of November-10th of December (3rd HD) acid solution $(A)$ and methanol $(B)$ and the time programme was as follow: 0' - 5\% B, 3' - 15\% B, $13^{\prime}-20 \%$ B, 25 ' $-25 \%$ B, 35' - 30\% B, 40 '- $35 \%$ B, $45^{\prime}-40 \%$ B, $47^{\prime}-45 \%$ B, $50^{\prime}-47 \%$ B, 60 ' - 48\% B, $64^{\prime}-50 \% \mathrm{~B}, 70^{\prime}-50 \% \mathrm{~B}, 75^{\prime}-5 \% \mathrm{~B}$. The flow rate was $0.85 \mathrm{~mL} / \mathrm{min}$, and the injection volume $40 \mu \mathrm{L}$. The total run time was 76 min. Individual phenols were quantified by a four-point regression curve on the basis of standards obtained from commercial suppliers. Individual phenols of olive paste were expressed as $\mathrm{mg} \mathrm{kg}^{-1}$.

Reference compounds: Oleuropein, verbascoside, and h-tyrosol were obtained from Extrasynthěse (Genay, France) and cinnamic acid, vanillic acid, p-coumaric acid, ferulic acid, apigenin, tyrosol, caffeic acid, luteolin, syringic acid, 3,4-dihydroxybenzoic acid, and taxifolin from Fluka (Steinheim, Germany), rutin, chlorogenic acid, 4-hydroxybiphenyl carboxylic acid, 4-hydroxyphenylacetic acid from Sigma-Aldrich (Steinheim, Germany).

\subsection{Total phenol content}

The total phenol content of methanolic extracts was analyzed using the modified isolation method described by Vasquez Roncero (Vasquez Roncero et al., 1973). The concentration of total polyphenols was estimated with Folin-Ciocalteau reagent at $725 \mathrm{~nm}$. The results were expressed as $\mathrm{mg}$ of gallic acid per $\mathrm{kg}$ of olive paste.

\subsection{DPPH radical scavenging activity}

The antioxidant activity was evaluated by measuring the radical scavenging effect of the fruits' methanolic extracts towards the 2,2-diphenyl1-picrylhydrazyl $\left(\mathrm{DPPH}^{\circ}\right)$ as reported previously by Singh et al., (2002). Five milliliters of a $0.1 \mathrm{mM}$ methanol solution of DPPH (Fluka) were added to $0.1 \mathrm{ml}$ of several concentrations of methanol extracts from the fruit samples. The tubes were allowed to stand at $27^{\circ} \mathrm{C}$ for $20 \mathrm{~min}$. The decrease in absorbance at $517 \mathrm{~nm}$ was recorded in a spectrophotometer (Shimadzu UV-vis mini spectrophotometer 1240). Radical scavenging activity was expressed as inhibition percentage and was calculated using the following formula: $\%$ Inhibition $=\left(\left[A_{\text {control }}-A_{\text {sample }}\right] / A_{\text {control }}\right) \times 100$ where $A_{\text {control }}=$ absorbance of the control reaction (containing all reagents except samples); $A_{\text {sample }}=$ absorbance of the test compound. $I_{50}$ in this test was defined as the concentration of the oil that was able to inhibit $50 \%$ of the total DPPH radicals. $I_{50}$ of the sample was expressed in $\mathrm{mg} / \mathrm{mL}$ and calculated through the interpolation of the linear regression analysis.

\subsection{Total Antioxidant Activity Assay}

Total antioxidant activity was determined using the ABTS method adapted from Miller and Rice-Evans (1997). Decolorization of the ABTS ${ }^{\circ+}$ radical cation by 
sample extract was measured at $734 \mathrm{~nm}$ in relation to a Trolox $®$ (6-hydroxy-2,5,7,8-tetramethylchroman2-carboxylic acid, Sigma-Aldrich) standard. Results

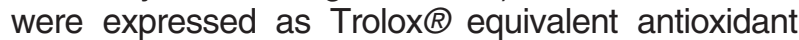
capacity (mmol TEAC/kg).

\subsection{Statistical analysis}

For the statistical analyses and evaluation of the experimental data, one-way analysis of variance (ONE-WAY ANOVA) was used to evaluate variety and season dependent differences regarding the parameters analyzed, while the t-test was used to evaluate year dependent differences. In the case of significance, differences between the mean values of specific varieties and seasons were evaluated using the Duncan's new multiple range test. All analyses were conducted using the statistical software program SPSS for Windows (v. 16). Significance was declared at $\mathrm{P} \leq 0.05$.

\section{RESULTS AND DISCUSSION}

\subsection{Phenolic Profiles of Olives}

An HPLC chromatogram of a fruit sample is reported as an example in Fig. 2. The phenolic composition of Sarıulak fruits is reported in Table 2. Among the 18 phenolic compounds oleuropein and h-tyrosol were identified as the main compounds, then in descending order tyrosol, rutin, verbascoside, 4-hydroxyphenyl acetic acid, luteolin, vanillic acid, 3,4-dihydroxybenzoic acid were detected and these were followed by apigenin, ferulic acid, chlorogenic acid, taxifolin, caffeic acid, syringic acid, p-coumaric acid, 4-hydroxybiphenyl carboxylic acid and cinnamic acid of which their amounts were under $40 \mathrm{mg} / \mathrm{kg}$ fresh fruit. Amiot et al., (1986), Servili et al., (1999) and Gomez-Rico et al., (2008) also reported that oleuropein showed the highest levels among the other phenolics in olive fruits, while Silva et al., (2006) reported h-tyrosol and rutin were the major phenolic compounds in olive fruits. The levels of most of the phenolic compounds in the fruits from the Alanya location were remarkably high among the values $(\mathrm{mg} / \mathrm{kg})$ obtained for all location samples mainly on the first HDs, for instance h-tyrosol rose to 3596.4 on the second HD in 2007, luteolin rose to 269.5 on the first HD in 2006, and taxifolin rose to 59.1 , caffeic acid rose to 62.1 , syringic acid to 60.8 , 4-hydroxybiphenyl carboxylic acid to 12.6 and vanillic acid rose to 159.8 on the first HDs in 2007. Together with the Alanya samples Karaman and Ceyhan fruits also contained higher amounts $(\mathrm{mg} / \mathrm{kg})$ of chlorogenic (0.1-76.6) and ferulic acids (8.2-56.9), respectively. Tyrosol was determined in a range of $11.9-872.4 \mathrm{mg} /$ $\mathrm{kg}$ and its level was lower in Karaman fruits than in Alanya, Silifke and Ceyhan fruits, whereas Karaman fruits showed the highest amounts of apigenin (2.1$79.8 \mathrm{mg} / \mathrm{kg}$ ) among these locations. The highest oleuropein, vanillic and cinnamic acid contents were detected for the fruit samples grown in Silifke and Karaman locations. Fruits from Silifke groves had the highest levels of verbascoside at concentrations of about 233.5 and $1107.5 \mathrm{mg} / \mathrm{kg}$, but Silifke fruits contained the lowest values of 3,4-dihydroxybenzoic acid among the other location fruits. 4-hydroxyphenyl acetic acid values ranged from 3.8 to $471.8 \mathrm{mg} / \mathrm{kg}$ and the lowest 4-hydroxyphenyl acetic acid values were determined in the Alanya fruits in 2006 and in Karaman samples in 2007. The fruits cultivated in Silifke were found to contain higher levels of h-tyrosol than the levels of fruits from other locations in 2006, while the highest levels were determined in the Alanya fruits in 2007.

The phenolic concentrations $(\mathrm{mg} / \mathrm{kg}$ ) determined in this study are closer to the previously reported results for some Portuguese and Spanish varieties in terms of rutin which were reported to vary between 66-500 (Gomez-Rico et al., 2008), 41.52114.80 (Vinha et al., 2002) and 175-1139 (in dry

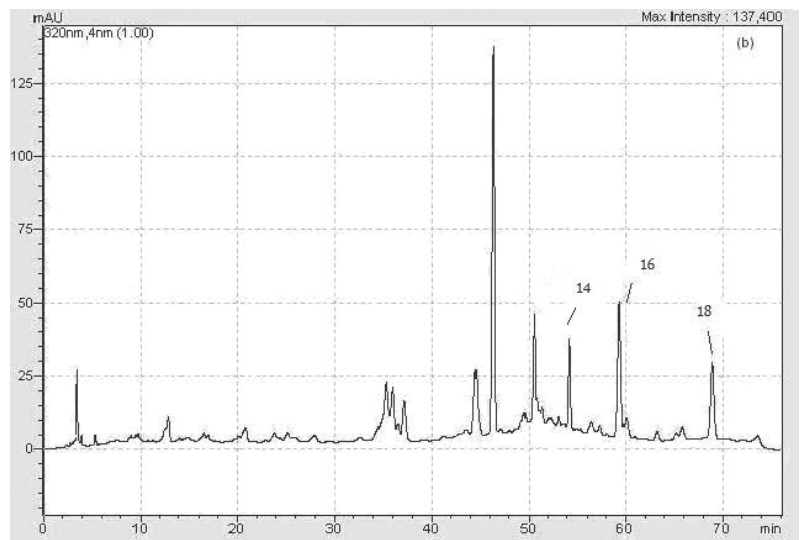

(1) Hydroxy tyrosol; (2) 3,4-dihydroxybenzoic acid; (3)tyrosol; (4) 4-hydroxyphenyl acetic acid; (5) chlorogenic acid; (6) vanillic acid; (7) caffeic acid; (8) syringic acid; (9) p-coumaric acid; (10) taxifolin; (11) ferulic acid; (12) verbascoside; (13) oleuropein; (14) rutin; (15) cinnamic acid; (16) luteolin; (17) 4-hyroxybiphenyl carboxylic acid; (18) apigenin. 
Table 2

Phenolic compounds in olive fruits of Sariulak variety $(\mathrm{mg} / \mathrm{kg})$

\begin{tabular}{|c|c|c|c|c|c|c|c|c|c|}
\hline \multirow{3}{*}{ Phenols } & \multirow{3}{*}{ H.D. ${ }^{\dagger}$} & \multicolumn{8}{|c|}{ Location } \\
\hline & & \multicolumn{2}{|c|}{ Alanya } & \multicolumn{2}{|c|}{ Karaman } & \multicolumn{2}{|c|}{ Ceyhan } & \multicolumn{2}{|c|}{ Silifke } \\
\hline & & 2006 & 2007 & 2006 & 2007 & 2006 & 2007 & 2006 & 2007 \\
\hline \multirow{3}{*}{$\begin{array}{c}\text { 4-H-biphenyl } \\
\text { carboxylic } \\
\text { acid }\end{array}$} & 1 & $5.9 \pm 1.5^{*} \mathrm{a}, \mathrm{A}, \mathrm{g}$ & $12.6 \pm 2.5 \mathrm{a}, \mathrm{A}, \mathrm{h}$ & $0.8 \pm 0.0 \mathrm{c}, \mathrm{A}, \mathrm{g}$ & $1.3 \pm 0.3 b, A, h$ & $1.2 \pm 0.2 b, A B$ & $1.5 \pm 0.2 b, A$ & $1.6 \pm 0.5 b, A$ & $1.2 \pm 0.2 b, A$ \\
\hline & 2 & $1.0 \pm 0.2 \mathrm{a}, \mathrm{B}, \mathrm{g}$ & $0.6 \pm 0.1 \mathrm{a}, \mathrm{B}, \mathrm{h}$ & $1.5 \pm 0.2 b, B, g$ & $2.8 \pm 0.8 \mathrm{c}, \mathrm{B}, \mathrm{h}$ & $0.4 \pm 0.0 \mathrm{c}, \mathrm{A}$ & $0.6 \pm 0.2 a, B$ & $2.4 \pm 0.2 \mathrm{~d}, \mathrm{~B}, \mathrm{~g}$ & $3.8 \pm 0.5 b, B, h$ \\
\hline & 3 & $1.8 \pm 0.2 a, C$ & $1.7 \pm 0.4 a, C$ & $0.4 \pm 0.1 b, \mathrm{C}, \mathrm{g}$ & $1.9 \pm 0.3 a, A B, h$ & $1.8 \pm 0.7 a, B$ & $11.3 \pm 18.3 a, A$ & $0.4 \pm 0.0 \mathrm{~b}, \mathrm{C}$ & $0.5 \pm 0.2 a, A$ \\
\hline \multirow{3}{*}{ Luteolin } & 1 & $269.5 \pm 33.5 \mathrm{c}, \mathrm{A}, \mathrm{g}$ & $4.2 \pm 0.8 \mathrm{a}, \mathrm{A}, \mathrm{h}$ & $38.9 \pm 7.3 \mathrm{a}, \mathrm{B}$ & $35.3 \pm 9.4 b c, A$ & $82.4 \pm 10.0 \mathrm{~d}, \mathrm{~A}, \mathrm{~g}$ & $55.2 \pm 15.0 \mathrm{c}, \mathrm{A}, \mathrm{h}$ & $210.5 \pm 43.5 b, A$ & $209.2 \pm 30.7 \mathrm{~d}, \mathrm{~A}$ \\
\hline & 2 & $127.6 \pm 11.9 \mathrm{a}, \mathrm{B}, \mathrm{g}$ & $37.4 \pm 8.6 \mathrm{~b}, \mathrm{~B}, \mathrm{~h}$ & $25.7 \pm 5.3 b, A$ & $17.3 \pm 2.5 \mathrm{a}, \mathrm{B}$ & $69.6 \pm 4.7 \mathrm{c}, \mathrm{A}, \mathrm{g}$ & $52.1 \pm 5.2 \mathrm{c}, \mathrm{A}, \mathrm{h}$ & $51.2 \pm 3.5 \mathrm{~d}, \mathrm{~B}, \mathrm{~g}$ & $29.9 \pm 3.4 b, B, h$ \\
\hline & 3 & $183.5 \pm 24.2 d, C$ & $225.7 \pm 27.0 d, C$ & $16.0 \pm 3.4 \mathrm{a}, \mathrm{A}, \mathrm{g}$ & $0.8 \pm 0.4 a, C, h$ & $140.7 \pm 17.4 c, \mathrm{~B}, \mathrm{~g}$ & $104.5 \pm 17.5 c, B, h$ & $38.4 \pm 7.1 b, \mathrm{C}, \mathrm{g}$ & $5.0 \pm 1.2 b, C, h$ \\
\hline \multirow{3}{*}{ Apigenin } & 1 & $54.8 \pm 9.7 \mathrm{a}, \mathrm{A}$ & $58.5 \pm 15.0 \mathrm{c}, \mathrm{A}$ & $79.8 \pm 11.3 b, A, g$ & $29.9 \pm 3.1 b, A, h$ & $1.3 \pm 0.1 \mathrm{c}, \mathrm{A}, \mathrm{g}$ & $3.3 \pm 0.6 \mathrm{a}, \mathrm{A}, \mathrm{h}$ & $3.8 \pm 1.2 \mathrm{~d}, \mathrm{~A}$ & $3.3 \pm 0.6 \mathrm{a}, \mathrm{A}$ \\
\hline & 2 & $3.4 \pm 0.9 \mathrm{~b}, \mathrm{~B}, \mathrm{~g}$ & $10.7 \pm 1.6 \mathrm{a}, \mathrm{B}, \mathrm{h}$ & $43.5 \pm 6.1 \mathrm{c}, \mathrm{B}, \mathrm{g}$ & $2.1 \pm 0.4 b, B, h$ & $28.0 \pm 3.0 \mathrm{a}, \mathrm{B}$ & $30.3 \pm 3.0 \mathrm{c}, \mathrm{B}$ & $46.8 \pm 7.1 \mathrm{c}, \mathrm{B}, \mathrm{g}$ & $28.2 \pm 3.6 \mathrm{c}, \mathrm{B}, \mathrm{h}$ \\
\hline & 3 & $1.6 \pm 0.3 c, C, g$ & $15.7 \pm 2.1 \mathrm{a}, \mathrm{C}, \mathrm{h}$ & $36.1 \pm 5.9 b, \mathrm{~B}, \mathrm{~g}$ & $2.6 \pm 0.5 b, \mathrm{~B}, \mathrm{~h}$ & $3.6 \pm 1.1 \mathrm{a}, \mathrm{C}, \mathrm{g}$ & $6.3 \pm 1.5 c, A, h$ & $4.3 \pm 1.4 a, A, g$ & $17.6 \pm 1.4 \mathrm{a}, \mathrm{C}, \mathrm{h}$ \\
\hline \multirow{3}{*}{ Rutin } & 1 & $141.8 \pm 12.8 \mathrm{a}, \mathrm{A}, \mathrm{g}$ & $206.7 \pm 20.0 \mathrm{~b}, \mathrm{~A}, \mathrm{~h}$ & $81.9 \pm 13.5 b, A, g$ & $63.2 \pm 5.6 \mathrm{a}, \mathrm{A}, \mathrm{h}$ & $22.9 \pm 4.0 \mathrm{c}, \mathrm{A}, \mathrm{g}$ & $66.1 \pm 7.7 \mathrm{a}, \mathrm{A}, \mathrm{h}$ & $55.2 \pm 7.7 \mathrm{~d}, \mathrm{~A}$ & $73.1 \pm 10.1 \mathrm{a}, \mathrm{A}$ \\
\hline & 2 & $199.8 \pm 15.8 \mathrm{a}, \mathrm{B}, \mathrm{g}$ & $232.9 \pm 13.6 \mathrm{c}, \mathrm{A}, \mathrm{h}$ & $105.9 \pm 10.6 \mathrm{~b}, \mathrm{~A}, \mathrm{~g}$ & $204.3 \pm 13.5$ b,B,h & $139.2 \pm 13.0 \mathrm{c}, \mathrm{B}$ & $124.0 \pm 8.4 \mathrm{a}, \mathrm{B}$ & $241.5 \pm 24.3 \mathrm{~d}, \mathrm{~B}, \mathrm{~g}$ & $192.5 \pm 13.5 b, B, h$ \\
\hline & 3 & $94.2 \pm 12.8 c, C, g$ & $242.8 \pm 39.8 a, A, h$ & $147.6 \pm 22.5 b, \mathrm{~B}$ & $48.9 \pm 77.0 \mathrm{~b}, \mathrm{~A}$ & $51.5 \pm 8.2 \mathrm{a}, \mathrm{C}, \mathrm{g}$ & $78.7 \pm 12.2 b, A, h$ & $39.8 \pm 11.9 \mathrm{a}, \mathrm{A}, \mathrm{g}$ & $83.4 \pm 9.3 b, A, h$ \\
\hline \multirow{3}{*}{ Verbascoside } & 1 & $22.0 \pm 5.0 \mathrm{c}, \mathrm{A}, \mathrm{g}$ & $255.1 \pm 10.6 \mathrm{a}, \mathrm{A}, \mathrm{h}$ & $39.4 \pm 9.8 \mathrm{a}, \mathrm{A}$ & $114.3 \pm 90.2 \mathrm{~b}, \mathrm{~A}$ & $78.4 \pm 30.7 \mathrm{a}, \mathrm{B}, \mathrm{g}$ & $561.6 \pm 33.4 \mathrm{c}, \mathrm{B}, \mathrm{h}$ & $242.9 \pm 51.5 b, A, g$ & $454.9 \pm 85.1 \mathrm{c}, \mathrm{A}, \mathrm{h}$ \\
\hline & 2 & $86.3 \pm 12.8 \mathrm{a}, \mathrm{B}, \mathrm{g}$ & $409.4 \pm 28.5 \mathrm{a}, \mathrm{B}, \mathrm{h}$ & $366.9 \pm 58.5 \mathrm{c}, \mathrm{B}, \mathrm{g}$ & $698.8 \pm 173.4 b, B, h$ & $43.6 \pm 12.7 \mathrm{~b}, \mathrm{AB}, \mathrm{g}$ & $484.1 \pm 58.3 \mathrm{ab}, \mathrm{B}, \mathrm{h}$ & $657.9 \pm 111.6 \mathrm{~d}, \mathrm{~B}$ & $1107.5 \pm 344.0 \mathrm{c}, \mathrm{B}$ \\
\hline & 3 & $23.4 \pm 6.4 \mathrm{a}, \mathrm{A}, \mathrm{g}$ & $151.4 \pm 42.9 a, C, h$ & $180.9 \pm 10.1 c, C$ & $168.5 \pm 43.1 \mathrm{a}, \mathrm{A}$ & $19.7 \pm 4.6$ a,A,g & $352.4 \pm 76.6 b, A, h$ & $233.5 \pm 26.6 b, A, g$ & $305.8 \pm 21.4 b, A, h$ \\
\hline \multirow{3}{*}{ Vanillic acid } & 1 & $15.6 \pm 0.6 \mathrm{~b}, \mathrm{~A}, \mathrm{~g}$ & $159.8 \pm 41.6 \mathrm{~d}, \mathrm{~A}, \mathrm{~h}$ & $46.6 \pm 4.8 \mathrm{c}, \mathrm{A}, \mathrm{h}$ & $18.2 \pm 1.9 b, A, g$ & $9.5 \pm 0.9 \mathrm{a}, \mathrm{A}, \mathrm{g}$ & $7.4 \pm 0.2 \mathrm{a}, \mathrm{A}, \mathrm{h}$ & $15.0 \pm 1.2 b, A, g$ & $48.1 \pm 4.8 \mathrm{c}, \mathrm{A}, \mathrm{h}$ \\
\hline & 2 & $54.6 \pm 5.8 \mathrm{~b}, \mathrm{~B}, \mathrm{~h}$ & $19.9 \pm 2.8 \mathrm{a}, \mathrm{B}, \mathrm{g}$ & $115.3 \pm 5.0 \mathrm{c}, \mathrm{B}, \mathrm{h}$ & $55.6 \pm 6.2 b, B, g$ & $14.6 \pm 1.2 \mathrm{a}, \mathrm{A}, \mathrm{g}$ & $21.8 \pm 3.6 \mathrm{a}, \mathrm{A}, \mathrm{h}$ & $52.8 \pm 4.5 \mathrm{~b}, \mathrm{~B}$ & $56.3 \pm 8.4 \mathrm{~b}, \mathrm{~A}$ \\
\hline & 3 & $20.1 \pm 2.5 \mathrm{a}, \mathrm{A}, \mathrm{g}$ & $81.2 \pm 9.9 a, C, h$ & $55.6 \pm 6.4 b, A, g$ & $94.8 \pm 10.4 a, C, h$ & $172.3 \pm 36.7 \mathrm{~d}, \mathrm{~B}, \mathrm{~g}$ & $77.1 \pm 12.1 \mathrm{a}, \mathrm{B}, \mathrm{h}$ & $78.8 \pm 9.7 c, C, g$ & $129.2 \pm 31.4 b, \mathrm{~B}, \mathrm{~h}$ \\
\hline \multirow{3}{*}{$\begin{array}{c}p \text {-Coumaric } \\
\text { acid }\end{array}$} & 1 & $12.8 \pm 1.3 \mathrm{a}, \mathrm{A}, \mathrm{g}$ & $24.4 \pm 1.6 \mathrm{a}, \mathrm{A}, \mathrm{h}$ & $4.5 \pm 0.5 b, A$ & $3.9 \pm 0.5 b, A$ & $23.6 \pm 3.4 \mathrm{c}, \mathrm{A}$ & $28.6 \pm 1.4 \mathrm{c}, \mathrm{A}$ & $1.4 \pm 0.3 \mathrm{~d}, \mathrm{~A}$ & $1.5 \pm 0.4 \mathrm{~d}, \mathrm{~A}$ \\
\hline & 2 & $16.4 \pm 1.8 \mathrm{a}, \mathrm{B}, \mathrm{g}$ & $23.8 \pm 1.3 \mathrm{a}, \mathrm{A}, \mathrm{h}$ & $0.1 \pm 0.0 \mathrm{c}, \mathrm{B}, \mathrm{g}$ & $0.5 \pm 0.0 \mathrm{~b}, \mathrm{~B}, \mathrm{~h}$ & $1.5 \pm 0.2 b, B$ & $1.5 \pm 0.3 \mathrm{c}, \mathrm{B}$ & $1.6 \pm 0.2 b, A$ & $1.7 \pm 0.2 \mathrm{c}, \mathrm{A}$ \\
\hline & 3 & $10.5 \pm 1.9 b, A, g$ & $16.0 \pm 2.8 c, B, h$ & $0.9 \pm 0.1 a, C$ & $0.9 \pm 0.2 a, C$ & $1.4 \pm 0.1 \mathrm{a}, \mathrm{B}$ & $1.3 \pm 0.0 a, B$ & $10.0 \pm 0.2 b, B$ & $10.9 \pm 1.0 \mathrm{~b}, \mathrm{~B}$ \\
\hline \multirow{3}{*}{ Ferulic acid } & 1 & $8.2 \pm 0.1 c, A, g$ & $56.9 \pm 8.1 \mathrm{c}, \mathrm{A}, \mathrm{h}$ & $21.5 \pm 2.6 \mathrm{a}, \mathrm{A}$ & $26.5 \pm 2.1 \mathrm{ab}, \mathrm{A}$ & $19.4 \pm 2.3 \mathrm{a}, \mathrm{A}$ & $19.2 \pm 2.3 \mathrm{a}, \mathrm{A}$ & $26.3 \pm 2.5 b, A, g$ & $32.1 \pm 2.9 \mathrm{~b}, \mathrm{~A}, \mathrm{~h}$ \\
\hline & 2 & $44.3 \pm 7.6 \mathrm{c}, \mathrm{B}, \mathrm{g}$ & $11.4 \pm 0.3 \mathrm{a}, \mathrm{B}, \mathrm{h}$ & $30.5 \pm 4.1 \mathrm{~b}, \mathrm{~B}, \mathrm{~g}$ & $9.8 \pm 0.3 \mathrm{a}, \mathrm{B}, \mathrm{h}$ & $39.0 \pm 4.5 b c, B, g$ & $30.2 \pm 4.5 \mathrm{~b}, \mathrm{~B}, \mathrm{~h}$ & $18.6 \pm 1.3 \mathrm{a}, \mathrm{B}, \mathrm{g}$ & $22.8 \pm 1.0 \mathrm{c}, \mathrm{B}, \mathrm{h}$ \\
\hline & 3 & $15.1 \pm 1.0 a, C$ & $34.6 \pm 35.4 a, A$ & $12.3 \pm 0.7 b, C, g$ & $9.2 \pm 0.1 \mathrm{a}, \mathrm{B}, \mathrm{h}$ & $15.6 \pm 0.2 a, A$ & $13.5 \pm 3.1 \mathrm{a}, \mathrm{A}$ & $9.5 \pm 0.2 c, \mathrm{C}, \mathrm{g}$ & $11.5 \pm 1.1 \mathrm{a}, \mathrm{C}, \mathrm{h}$ \\
\hline \multirow{3}{*}{ Caffeic acid } & 1 & $26.9 \pm 3.9 b, A, g$ & $62.1 \pm 2.1 \mathrm{~d}, \mathrm{~A}, \mathrm{~h}$ & $0.7 \pm 0.2 \mathrm{a}, \mathrm{A}$ & $0.8 \pm 0.1 \mathrm{a}, \mathrm{A}$ & $15.8 \pm 0.6 \mathrm{c}, \mathrm{A}, \mathrm{g}$ & $14.7 \pm 0.2 c, A, h$ & $1.3 \pm 0.2 \mathrm{a}, \mathrm{A}, \mathrm{g}$ & $0.5 \pm 0.0 \mathrm{~b}, \mathrm{~A}, \mathrm{~h}$ \\
\hline & 2 & $12.1 \pm 1.3 \mathrm{c}, \mathrm{B}, \mathrm{g}$ & $16.5 \pm 0.2 \mathrm{c}, \mathrm{B}, \mathrm{h}$ & $1.2 \pm 0.0 \mathrm{a}, \mathrm{B}$ & $1.2 \pm 0.1 \mathrm{a}, \mathrm{B}$ & $1.6 \pm 0.0 \mathrm{a}, \mathrm{B}, \mathrm{g}$ & $1.3 \pm 0.1 \mathrm{a}, \mathrm{B}, \mathrm{h}$ & $6.3 \pm 0.2 b, B, g$ & $4.6 \pm 0.1 \mathrm{~b}, \mathrm{~B}, \mathrm{~h}$ \\
\hline & 3 & $3.3 \pm 0.2 a, C, g$ & $0.2 \pm 0.0 a, C, h$ & $7.2 \pm 0.1 b, C, g$ & $3.7 \pm 0.1 b, \mathrm{C}, \mathrm{h}$ & $2.0 \pm 0.4 c, C, g$ & $4.3 \pm 0.6 b, C, h$ & $0.3 \pm 0.0 \mathrm{~d}, \mathrm{C}, \mathrm{g}$ & $0.8 \pm 0.2 a, C, h$ \\
\hline & 1 & $1.9 \pm 1.1 \mathrm{a}, \mathrm{AB}, \mathrm{g}$ & $0.4 \pm 0.0 \mathrm{a}, \mathrm{A}, \mathrm{h}$ & $4.1 \pm 0.4 \mathrm{~b}, \mathrm{~A}, \mathrm{~g}$ & $1.3 \pm 0.4 \mathrm{~b}, \mathrm{~A}, \mathrm{~h}$ & $1.9 \pm 0.4 \mathrm{a}, \mathrm{A}, \mathrm{g}$ & $1.2 \pm 0.3 b, B, h$ & $4.7 \pm 1.0 \mathrm{~b}, \mathrm{~A}, \mathrm{~g}$ & $1.2 \pm 0.3 b, B, h$ \\
\hline Cinnamic acid & 2 & $2.4 \pm 0.4 \mathrm{a}, \mathrm{A}, \mathrm{g}$ & $0.3 \pm 0.1 \mathrm{a}, \mathrm{A}, \mathrm{h}$ & $5.9 \pm 1.3 b, B, g$ & $0.2 \pm 0.0 \mathrm{a}, \mathrm{B}, \mathrm{h}$ & $0.6 \pm 0.1 \mathrm{a}, \mathrm{B}, \mathrm{g}$ & $1.0 \pm 0.2 b, B, h$ & $13.6 \pm 3.9 \mathrm{c}, \mathrm{B}, \mathrm{g}$ & $1.4 \pm 0.3 \mathbf{c}, \mathrm{B}, \mathrm{h}$ \\
\hline & 3 & $0.9 \pm 0.1 \mathrm{a}, \mathrm{B}, \mathrm{g}$ & $2.2 \pm 0.4 a, B, h$ & $5.3 \pm 0.4 c, A B, g$ & $0.5 \pm 0.1 b, \mathrm{~B}, \mathrm{~h}$ & $1.2 \pm 0.3 a, C, g$ & $0.4 \pm 0.4 b, A, h$ & $0.5 \pm 0.0 \mathrm{~b}, \mathrm{C}$ & $0.6 \pm 0.1 b, A$ \\
\hline & 1 & $644.2 \pm 55.2 \mathrm{a}, \mathrm{A}, \mathrm{g}$ & $933.3 \pm 120.5 b c, A, h$ & $1014.9 \pm 161.9 \mathrm{~b}, \mathrm{~A}$ & $807.3 \pm 76.4 \mathrm{~b}, \mathrm{~A}$ & $426.8 \pm 52.7 \mathrm{a}, \mathrm{A}, \mathrm{g}$ & $631.9 \pm 48.9 \mathrm{a}, \mathrm{A}, \mathrm{h}$ & $1222.7 \pm 197.5 \mathrm{~b}, \mathrm{~A}, \mathrm{~g}$ & $963.5 \pm 50.2 c, A, h$ \\
\hline Oleuropein & 2 & $1141.5 \pm 685.6$ b,A & $1102.8 \pm 49.5 \mathrm{a}, \mathrm{A}$ & $1071.8 \pm 102.4 b, A$ & $1104.3 \pm 113.9 \mathrm{a}, \mathrm{B}$ & $1697.0 \pm 176.7$ b,B,g & $1166.8 \pm 127.5 \mathrm{a}, \mathrm{B}, \mathrm{h}$ & $2981.8 \pm 384.4 \mathrm{a}, \mathrm{B}$ & $2487.2 \pm 339.30 \mathrm{~b}, \mathrm{~B}$ \\
\hline & 3 & $254.5 \pm 1.6 c, B, g$ & $413.5 \pm 79.4 \mathrm{a}, \mathrm{B}, \mathrm{h}$ & $835.2 \pm 185.1 a, A$ & $655.6 \pm 99.1 b, A$ & $290.2 \pm 17.7 \mathrm{~b}, \mathrm{C}, \mathrm{g}$ & $340.4 \pm 9.0 a, C, h$ & $375.8 \pm 87.1 b, c$ & $427.2 \pm 33.7 a, C$ \\
\hline & 1 & $49.2 \pm 16.5 \mathrm{a}, \mathrm{A}, \mathrm{g}$ & $872.4 \pm 88.6$ b,A,h & $154.1 \pm 30.5 c, A, g$ & $106.7 \pm 14.3 \mathrm{a}, \mathrm{A}, \mathrm{h}$ & $93.3 \pm 16.5 b, A$ & $128.3 \pm 33.3 \mathrm{a}, \mathrm{A}$ & $173.0 \pm 38.8 \mathrm{c}, \mathrm{B}, \mathrm{g}$ & $87.4 \pm 11.3 \mathrm{a}, \mathrm{h}$ \\
\hline Tyrosol & 2 & $68.9 \pm 8.3 \mathrm{a}, \mathrm{A}, \mathrm{g}$ & $766.8 \pm 73.7 \mathrm{c}, \mathrm{A}, \mathrm{h}$ & $47.5 \pm 9.6 \mathrm{a}, \mathrm{B}, \mathrm{g}$ & $20.1 \pm 3.4 \mathrm{a}, \mathrm{B}, \mathrm{h}$ & $49.9 \pm 1.9 \mathrm{a}, \mathrm{B}, \mathrm{g}$ & $86.8 \pm 11.3 b, A, h$ & $210.1 \pm 22.7 b, B$ & $188.7 \pm 10.0 \mathrm{~d}$ \\
\hline & 3 & $103.2 \pm 12.7 \mathrm{a}, \mathrm{B}, \mathrm{g}$ & $241.2 \pm 34.1 \mathrm{c}, \mathrm{B}, \mathrm{h}$ & $126.4 \pm 2.6 c, A, g$ & $78.9 \pm 5.0 \mathrm{a}, \mathrm{C}, \mathrm{h}$ & $142.0 \pm 15.2 c, C$ & $194.6 \pm 39.9 c, B$ & $27.1 \pm 7.8 \mathrm{~b}, \mathrm{~A}, \mathrm{~g}$ & $11.9 \pm 2.7 b, \mathrm{~h}$ \\
\hline & 1 & $11.3 \pm 1.1 \mathrm{a}, \mathrm{A}, \mathrm{g}$ & $59.1 \pm 9.4 \mathrm{~d}, \mathrm{~B}, \mathrm{~h}$ & $9.1 \pm 0.3 b, A, g$ & $11.7 \pm 1.6 \mathrm{a}, \mathrm{A}, \mathrm{h}$ & $10.7 \pm 0.4 \mathrm{a}, \mathrm{A}, \mathrm{g}$ & $18.4 \pm 2.3 c, B, h$ & $2.3 \pm 0.3 c, A, g$ & $3.6 \pm 0.3 b, A, h$ \\
\hline Taxifolin & 2 & $7.5 \pm 2.2 \mathrm{a}, \mathrm{B}, \mathrm{g}$ & $12.7 \pm 2.3 \mathrm{a}, \mathrm{A}, \mathrm{h}$ & $4.8 \pm 0.7 \mathrm{a}, \mathrm{B}, \mathrm{g}$ & $1.3 \pm 0.2 b, B, h$ & $8.2 \pm 1.0 \mathrm{a}, \mathrm{B}$ & $7.5 \pm 1.7 \mathrm{c}, \mathrm{A}$ & $15.0 \pm 3.5 b, B$ & $13.8 \pm 1.6 \mathrm{a}, \mathrm{B}$ \\
\hline & 3 & $1.3 \pm 0.3 \mathrm{a}, \mathrm{C}, \mathrm{g}$ & $18.4 \pm 3.3 a, A, h$ & $6.0 \pm 0.4 c, C, g$ & $4.3 \pm 0.2 b, C, h$ & $5.4 \pm 0.3 b, C, g$ & $4.1 \pm 0.8 b, A, h$ & $1.5 \pm 0.2 \mathrm{a}, \mathrm{A}, \mathrm{g}$ & $2.0 \pm 0.2 c, C, h$ \\
\hline & 1 & $427.6 \pm 59.3 \mathrm{a}, \mathrm{A}, \mathrm{g}$ & $1280.2 \pm 3.0 \mathrm{a}, \mathrm{A}, \mathrm{h}$ & $204.7 \pm 25.7 \mathrm{~b}, \mathrm{~A}, \mathrm{~g}$ & $47.3 \pm 6.8 b, A, h$ & $95.8 \pm 14.2 c, A, g$ & $418.5 \pm 1.5 \mathrm{c}, \mathrm{A}, \mathrm{h}$ & $563.2 \pm 69.6 \mathrm{~d}, \mathrm{~A}, \mathrm{~g}$ & $126.7 \pm 0.2 \mathrm{~d}, \mathrm{~A}, \mathrm{~h}$ \\
\hline H-tyrosol & 2 & $212.6 \pm 17.9 \mathrm{~b}, \mathrm{~B}, \mathrm{~g}$ & $3596.4 \pm 628.0 \mathrm{c}, \mathrm{B}, \mathrm{h}$ & $298.8 \pm 43.5 b, A, g$ & $73.0 \pm 0.0 \mathrm{a}, \mathrm{B}, \mathrm{h}$ & $86.1 \pm 15.6 \mathrm{a}, \mathrm{A}, \mathrm{g}$ & $394.9 \pm 91.7 \mathrm{~b}, \mathrm{~A}, \mathrm{~h}$ & $1499.1 \pm 104.8 \mathbf{c}, \mathrm{B}, \mathrm{g}$ & $550.4 \pm 74.5 \mathrm{~b}, \mathrm{~B}, \mathrm{~h}$ \\
\hline & 3 & $38.7 \pm 5.9 \mathrm{a}, \mathrm{C}, \mathrm{g}$ & $546.2 \pm 13.2 \mathrm{c}, \mathrm{C}, \mathrm{h}$ & $525.2 \pm 71.3 \mathrm{~b}, \mathrm{~B}, \mathrm{~g}$ & $135.2 \pm 4.2 \mathrm{a}, \mathrm{C}, \mathrm{h}$ & $499.4 \pm 85.4 b, \mathrm{~B}$ & $557.3 \pm 58.9 c, B$ & $675.3 \pm 130.7 c, A, g$ & $412.0 \pm 87.5 b, \mathrm{~B}, \mathrm{~h}$ \\
\hline & 1 & $14.4 \pm 3.6 \mathrm{a}, \mathrm{A}$ & $20.2 \pm 1.9 \mathrm{c}, \mathrm{A}$ & $1.5 \pm 0.2 b, A$ & $1.5 \pm 0.1 \mathrm{~b}, \mathrm{~A}$ & $0.0 \pm 0.0 \mathrm{c}, \mathrm{A}$ & $0.0 \pm 0.0 a, A$ & $23.3 \pm 2.3 \mathrm{~d}, \mathrm{~A}$ & $25.0 \pm 18.6 c, A$ \\
\hline llorogenic & 2 & $44.1 \pm 1.7 \mathrm{a}, \mathrm{B}, \mathrm{g}$ & $63.3 \pm 2.3 \mathrm{c}, \mathrm{B}, \mathrm{h}$ & $37.9 \pm 4.6 \mathrm{~b}, \mathrm{~B}$ & $39.5 \pm 4.7 \mathrm{~b}, \mathrm{~B}$ & $0.0 \pm 0.0 \mathrm{c}, \mathrm{A}$ & $0.0 \pm 0.0 \mathrm{a}, \mathrm{A}$ & $0.0 \pm 0.0 \mathrm{c}, \mathrm{B}$ & $0.0 \pm 0.0 \mathrm{a}, \mathrm{B}$ \\
\hline & 3 & $0.3 \pm 0.1 \mathrm{a}, \mathrm{C}$ & $0.1 \pm 0.1 b, c$ & $45.5 \pm 7.8 b, \mathrm{~B}, \mathrm{~g}$ & $76.6 \pm 5.8 c, C, h$ & $1.5 \pm 0.2 \mathrm{~d}, \mathrm{~B}, \mathrm{~g}$ & $1.1 \pm 0.1 b, \mathrm{~B}, \mathrm{~h}$ & $0.0 \pm 0.0 c, B$ & $0.0 \pm 0.0 \mathrm{a}, \mathrm{B}$ \\
\hline & 1 & $128.4 \pm 26.5 \mathrm{a}, \mathrm{B}, \mathrm{g}$ & $427.4 \pm 53.0 \mathrm{a}, \mathrm{A}, \mathrm{h}$ & $158.1 \pm 26.4 \mathrm{a}, \mathrm{A}, \mathrm{g}$ & $84.2 \pm 20.6 \mathrm{~b}, \mathrm{~A}, \mathrm{~h}$ & $313.1 \pm 48.0 \mathrm{~b}, \mathrm{~B}, \mathrm{~g}$ & $103.6 \pm 19.2 \mathrm{~b}, \mathrm{~A}, \mathrm{~h}$ & $239.5 \pm 24.8 \mathrm{c}, \mathrm{A}, \mathrm{g}$ & $48.3 \pm 3.8 \mathrm{c}, \mathrm{A}, \mathrm{h}$ \\
\hline onenylacetic & 2 & $12.2 \pm 2.0 \mathrm{a}, \mathrm{A}, \mathrm{g}$ & $172.9 \pm 37.4 \mathrm{~d}, \mathrm{~B}, \mathrm{~h}$ & $166.0 \pm 52.6 \mathrm{c}, \mathrm{A}, \mathrm{g}$ & $10.1 \pm 1.1 \mathrm{a}, \mathrm{B}, \mathrm{h}$ & $74.4 \pm 9.2 b, A$ & $49.6 \pm 14.3 b, C$ & $471.8 \pm 55.9 \mathrm{~d}, \mathrm{~B}, \mathrm{~g}$ & $332.7 \pm 32.1 \mathrm{c}, \mathrm{B}, \mathrm{h}$ \\
\hline & 3 & $102.6 \pm 12.1 \mathrm{a}, \mathrm{B}, \mathrm{g}$ & $19.1 \pm 3.5 \mathrm{a}, \mathrm{C}, \mathrm{h}$ & $225.6 \pm 45.6 b, A, g$ & $47.8 \pm 9.3 b, C, h$ & $335.4 \pm 37.7 \mathrm{c}, \mathrm{B}, \mathrm{g}$ & $224.8 \pm 44.3 c, B, h$ & $3.8 \pm 0.3 d, C, g$ & $21.7 \pm 2.8 \mathrm{a}, \mathrm{C}, \mathrm{h}$ \\
\hline & 1 & $7.9 \pm 0.4 \mathrm{a}, \mathrm{A}, \mathrm{g}$ & $90.7 \pm 2.9 \mathrm{c}, \mathrm{A}, \mathrm{h}$ & $81.2 \pm 9.6 b, A B, g$ & $54.1 \pm 4.8 \mathrm{~b}, \mathrm{~A}, \mathrm{~h}$ & $39.4 \pm 1.9 \mathrm{c}, \mathrm{A}, \mathrm{g}$ & $33.7 \pm 1.2 \mathrm{a}, \mathrm{A}, \mathrm{h}$ & $103.9 \pm 8.8 \mathrm{~d}, \mathrm{~A}, \mathrm{~g}$ & $56.7 \pm 6.8 b, A, h$ \\
\hline 3-4 H-benzolc & 2 & $3.6 \pm 0.7 \mathrm{a}, \mathrm{B}, \mathrm{g}$ & $172.2 \pm 5.0 \mathrm{c}, \mathrm{B}, \mathrm{h}$ & $73.5 \pm 2.3 \mathrm{c}, \mathrm{A}, \mathrm{g}$ & $25.3 \pm 1.8 \mathrm{a}, \mathrm{B}, \mathrm{h}$ & $56.8 \pm 6.3 b, B, g$ & $42.8 \pm 3.8 b, B, h$ & $69.3 \pm 1.8 \mathrm{c}, \mathrm{B}, \mathrm{g}$ & $45.5 \pm 2.0 \mathrm{~b}, \mathrm{~B}, \mathrm{~h}$ \\
\hline & 3 & $14.0 \pm 0.3 \mathrm{a}, \mathrm{C}, \mathrm{g}$ & $74.7 \pm 3.5 \mathrm{a}, \mathrm{C}, \mathrm{h}$ & $90.6 \pm 5.8 b, \mathrm{~B}, \mathrm{~g}$ & $24.8 \pm 0.4 b, \mathrm{~B}, \mathrm{~h}$ & $103.6 \pm 2.6 c, C, g$ & $74.2 \pm 4.1 \mathrm{a}, \mathrm{C}, \mathrm{h}$ & $43.0 \pm 2.9 \mathrm{~d}, \mathrm{C}, \mathrm{g}$ & $25.8 \pm 2.5 b, C, h$ \\
\hline & 1 & $27.8 \pm 0.8 \mathrm{a}, \mathrm{A}, \mathrm{g}$ & $60.8 \pm 5.2 c, A, h$ & $0.3 \pm 0.1 b, A, g$ & $0.0 \pm 0.0 \mathrm{a}, \mathrm{A}, \mathrm{h}$ & $0.3 \pm 0.1 \mathrm{~b}, \mathrm{~A}, \mathrm{~g}$ & $0.8 \pm 0.2 \mathrm{~b}, \mathrm{~A}, \mathrm{~h}$ & $1.8 \pm 0.3 c, A, g$ & $1.2 \pm 0.1 b, A, h$ \\
\hline Syringic acid & 2 & $0.7 \pm 0.1 \mathrm{a}, \mathrm{B}, \mathrm{g}$ & $1.1 \pm 0.1 \mathrm{a}, \mathrm{B}, \mathrm{h}$ & $2.4 \pm 0.2 b, B, g$ & $0.7 \pm 0.1 b, B, h$ & $1.6 \pm 0.1 \mathrm{c}, \mathrm{B}, \mathrm{g}$ & $2.3 \pm 0.3 c, B, h$ & $4.0 \pm 0.4 \mathrm{~d}, \mathrm{~B}, \mathrm{~g}$ & $3.3 \pm 0.2 \mathrm{~d}, \mathrm{~B}, \mathrm{~h}$ \\
\hline & 3 & $3.7 \pm 0.7 b, c$ & $3.9 \pm 0.3 c, C$ & $2.5 \pm 0.2 c, B, g$ & $0.5 \pm 0.0 b, \mathrm{C}, \mathrm{h}$ & $3.4 \pm 0.5 b, C$ & $2.8 \pm 0.5 \mathrm{~d}, \mathrm{~B}$ & $0.2 \pm 0.0 \mathrm{a}, \mathrm{C}, \mathrm{g}$ & $0.0 \pm 0.0 \mathrm{a}, \mathrm{C}, \mathrm{h}$ \\
\hline
\end{tabular}

*mean value \pm standard deviation

†H.D.:harvest date

$a, b, c, d, e$ : Mean values of the same harvest date and the same crop year with a different superscript differ significantly $(P \leq 0.05)$ [comparison between varieties]

$A, B, C$ : Mean values of the same variety and the same crop year with a different superscript differ significantly $(P \leq 0.05)$ [comparison between harvest dates]

$\mathrm{g}, \mathrm{h}$ : Mean values of the same variety and the same harvest date with a different superscript differ significantly $(\mathrm{P} \leq 0.05)$ [comparison between crop years]

The normal, bold and italic small letters refer to the first, second and third harvest dates, respectively. 
matter) (Artajo et al., 2006), luteolin 96.51-136.39 (Artajo et al., 2006), 3.3-440 (in dry matter) (Vinha et al., 2005), 62-529 (Gomez-Rico et al., 2008), verbascoside $0-1231 \mathrm{mg} / \mathrm{kg}$ (Gomez-Rico et al., 2008) and oleuropein 388-21681 (in dry matter). Apigenin, h-tyrosol, tyrosol and vanillic acid were found to be present at higher levels in Sarıulak variety fruits in this study than the values reported by Artajo et al. (2006) for the Spanish variety Arbequina as 4.65-6.09, 93.77-161.04, 82.84-92.59 and $12.58-18.29 \mathrm{mg} / \mathrm{kg}$ for the mentioned phenolic compounds, respectively.

Romero et al. (2003) investigated the composition of virgin olive oils produced over four consecutive crop seasons, taking the harvest period and the climatic conditions into consideration and found that phenolic profiles were mainly influenced by the cumulative rainfall. Romero et al. (2003), Tovar et al. (2002) and Yousfi et al. (2006) reported that there were reductions in phenolic concentrations of olive oils from fruits of olive trees cultivated in irrigated areas or under high rainfall conditions when compared to the oils from non-irrigated or low rainfall areas. On the other hand, a positive relationship was reported between phenol content and precipitation for the virgin olive oils from the Chetoi variety (Ben Temime et al., 2006). As can be seen from Figure 1, the Alanya location got the highest average rainfall among the locations studied and the Karaman location is at the highest average altitude. The higher levels of phenolic compounds in the Alanya samples in this study do not corroborate the correlation noticed by Tovar et al. (2002), Romero et al. (2003) and Yousfi et al. (2006), but agrees with the results of Ben Temime et al. (2006). The olives that were cultivated in higher altitude locations were reported to contain higher percentages of phenolics than the olives from lower altitude locations (Mousa et al., 1996). Our observations do not confirm this effect, as the fruits cultivated in Karaman did not show the highest phenolic levels.

Several agronomic parameters such as cultivar and geographical origin, fruit ripening, harvest period and climate conditions modify the phenolic composition of olives (Esti et al., 1998; Botía et al., 2001). Vinha et al. (2005) reported that although the strict influence of one factor can only be evaluated when all other factors remain constant, some clear conclusions can be drawn, for example, samples from the same cultivar but with different maturation index and geographical origins have similar phenolic profiles for the major compounds, pointing to a strong influence on the part of the cultivar. However, when comparing the samples from the same geographical origin and with similar maturation index, but collected from different cultivars, they showed very similar phenolic profiles, denoting a strong influence of geographical origin.

When comparing the results by harvest time, significant differences can be seen between the three dates in terms of the phenolic compounds of the olive fruits. Rutin, verbascoside, oleuropein and in most cases taxifolin and tyrosol showed the highest levels on the second HDs but then decreased on the third HDs in all the samples. Apigenin, cinnamic acid and in most cases p-coumaric and 4-hydroxybiphenyl carboxylic acids levels decreased as the HD progressed, while on the contrary, ferulic and vanillic acid levels increased in all location samples with progressing HD. The effect of HD on the rest of the phenolic compounds identified in this work was different in relation to the location. The olives cultivated in Silifke contained higher levels of 4-hydroxyphenyl acetic acid, caffeic acid, syringic acid and h-tyrosol on the second HD when compared to first and third HDs. Luteolin, chlorogenic acid and 3,4-dihydroxybenzoic acid levels of Silifke samples decreased as the HD progressed. The remaining phenolics in olives from Alanya decreased except luteolin which showed an increasing trend, whereas the values of these phenolics except caffeic acid increased in Ceyhan samples towards later HDs. Luteolin, 3,4-dihydroxybenzoic acid and 4-hydroxyphenyl acetic acid levels decreased while caffeic acid, syringic acid, chlorogenic acid and h-tyrosol values increased in the fruits cultivated in Karaman in later harvested fruits. There are studies on the phenolic contents of olive fruits which report that there were clear increases in the concentrations of h-tyrosol, verbascoside, rutin and luteolin and decreases in the concentrations of oleuropein with ripeness or harvest time (Gomez-Rico et al., 2008; Artajo et al., 2006; Ryan et al., 1999) which is in accordance with the changes in oleuropein, h-tyrosol (for Karaman and Ceyhan locations) and luteolin (Alanya and Ceyhan locations) in this study. But, rutin and verbascoside concentrations decreased towards the last harvest after an increase on the second harvest date unlike the results of Gomez-Rico et al. (2008) and Artajo et al. (2006). In the case of tyrosol, our results for some varieties do not confirm what was reported by Boskou (2006) and Ryan et al. (1999) where the authors claimed that tyrosol content increases during the ripening of olive fruits. Nonetheless, Ryan et al. (1999) reported that there was a decrease in the tyrosol content of the Cucco variety during the course of ripening and then an increase through the black stage of maturation, while there was no significant change in tyrosol for the Manzanilla variety. However, our findings are in agreement with the results of Boskou (2006) and Ryan et al. (1999) in the case of oleuropein and h-tyrosol as they reported that the oleuropein content decreases and tyrosol and h-tyrosol (for Karaman and Ceyhan locations) contents increase with the ripening of olive fruits. In the case of verbascoside, Ryan et al. (1999) reported similar results as the content of this phenolic compound decreases when the HD progresses.

The changes in verbascoside, rutin, luteolin, cinnamic acid, 4-hydroxyphenyl acetic acid and 4-hydroxybiphenyl carboxylic acid according to crop year appeared to be less dependent on the cultivation area. The first two phenolic compounds showed increasing trends in all the samples in 
Table 3

Antioxidant activity, radical scavenging effect and total phenolic content of olive fruits from Sariulak variety

\begin{tabular}{|c|c|c|c|c|c|c|c|}
\hline \multirow[t]{2}{*}{ Location } & \multirow[t]{2}{*}{ H.D. ${ }^{\dagger}$} & \multicolumn{2}{|c|}{$\begin{array}{l}\text { Antioxidant activity } \\
\text { (TEAC, mmol TE/kg) }\end{array}$} & \multicolumn{2}{|c|}{$\begin{array}{l}\text { Radical scavenging activity } \\
\text { (DPPH, IC }{ }_{50} \text { ) }\end{array}$} & \multicolumn{2}{|c|}{$\begin{array}{c}\text { Total phenolics } \\
\text { (mg Gallic acid/ kg fresh fruit) }\end{array}$} \\
\hline & & 2006 & 2007 & 2006 & 2007 & 2006 & 2007 \\
\hline \multirow{3}{*}{ Alanya } & 1 & $3.1 \pm 0.0^{\star} a, A, g$ & $1.6 \pm 0.1 \mathrm{a}, \mathrm{A}, \mathrm{h}$ & $0.68 \pm 0.07 \mathrm{a}, \mathrm{A}, \mathrm{g}$ & $0.45 \pm 0.04 \mathrm{a}, \mathrm{B}, \mathrm{h}$ & $275.8 \pm 47.4 \mathrm{a}, \mathrm{A}, \mathrm{g}$ & $424.9 \pm 2$ \\
\hline & 2 & $1.7 \pm 0.2 \mathrm{a}, \mathrm{B}, \mathrm{h}$ & $2.3 \pm 0.2 \mathrm{a}, \mathrm{B}, \mathrm{g}$ & $0.67 \pm 0.01 \mathrm{a}, \mathrm{A}, \mathrm{g}$ & $0.32 \pm 0.02 \mathrm{a}, \mathrm{A}, \mathrm{h}$ & $288.1 \pm 61.2 \mathrm{a}, \mathrm{A}, \mathrm{g}$ & $476.0 \pm 47.6 \mathrm{a}, \mathrm{A}, \mathrm{h}$ \\
\hline & 3 & $1.2 \pm 0.3 a, \mathrm{C}, \mathrm{h}$ & $2.2 \pm 0.2 b, \mathrm{~B}, \mathrm{~g}$ & $1.05 \pm 0.09 a, B$ & $0.99 \pm 0.10 a, C$ & $203.5 \pm 40.6 a, A, g$ & $405.7 \pm 41.9 a, A, h$ \\
\hline \multirow{3}{*}{ Karaman } & r & $2.1 \pm 0.1 b, A, g$ & $1.7 \pm 0.1 \mathrm{a}, \mathrm{A}, \mathrm{h}$ & $0.64 \pm 0.03 a, A, h$ & $1.02 \pm 0.06 b$ & $32.2 \pm 88$ & $394.1 \pm 6$ \\
\hline & 2 & $2.3 \pm 0.1 \mathrm{~b}, \mathrm{~A}$ & $2.2 \pm 0.2 \mathrm{a}, \mathrm{B}$ & $0.75 \pm 0.01 \mathrm{a}, \mathrm{C}, \mathrm{h}$ & $1.36 \pm 0.16$ b,B,g & $345.5 \pm 33.5 \mathrm{ab}, \mathrm{A}$ & $396.0 \pm 42.7 \mathrm{~b}, \mathrm{~A}$ \\
\hline & 3 & $2.9 \pm 0.3 b, \mathrm{~B}, \mathrm{~g}$ & $1.8 \pm 0.3 a, A, h$ & $0.43 \pm 0.02 b, \mathrm{~B}, \mathrm{~h}$ & $1.31 \pm 0.06 b, \mathrm{~B}, \mathrm{~g}$ & $337.0 \pm 67.1 b, \mathrm{~A}$ & $386.7 \pm 69.2 a, A$ \\
\hline \multirow{3}{*}{ Ceyhan } & 1 & $1.4 \pm 0.1 \mathrm{~d}, \mathrm{Ag}$ & $0.9 \pm 0.2 b, A, h$ & $1.32 \pm 0.09 \mathrm{c}, \mathrm{A}, \mathrm{g}$ & $0.75 \pm 0.01 \mathrm{c}, \mathrm{B}, \mathrm{h}$ & $227.3 \pm 40.3 \mathrm{a}, \mathrm{AB}, \mathrm{g}$ & $342.5 \pm 54.2 \mathrm{a}, \mathrm{A}, \mathrm{h}$ \\
\hline & 2 & $1.6 \pm 0.2 \mathrm{a}, \mathrm{Ag}$ & $1.3 \pm 0.1 b, B, h$ & $1.14 \pm 0.08$ b,B,g & $0.62 \pm 0.05 \mathrm{c}, \mathrm{A}, \mathrm{h}$ & $275.5 \pm 34.1 \mathrm{a}, \mathrm{B}, \mathrm{g}$ & $380.8 \pm 48.1 \mathrm{~b}, \mathrm{~A}, \mathrm{~h}$ \\
\hline & 3 & $2.5 \pm 0.3 b, \mathrm{~B}$ & $2.2 \pm 0.2 b, c$ & $0.88 \pm 0.10 c, \mathrm{C}, \mathrm{g}$ & $0.57 \pm 0.02 c, A, h$ & $180.5 \pm 47.0 a, A, g$ & $423.3 \pm 3.1 \mathrm{a}, \mathrm{B}, \mathrm{h}$ \\
\hline \multirow{3}{*}{ Silifke } & 1 & $2.4 \pm 0.1 \mathrm{c}, \mathrm{A}, \mathrm{g}$ & $1.7 \pm 0.1 \mathrm{a}, \mathrm{A}, \mathrm{h}$ & $0.81 \pm 0.01 b, B, h$ & $1.46 \pm 0.02 \mathrm{~d}, \mathrm{~B}, \mathrm{~g}$ & $322.9 \pm 56.8 \mathrm{a}, \mathrm{AB}$ & $341.3 \pm 9.1 \mathrm{a}, \mathrm{A}$ \\
\hline & 2 & $3.2 \pm 0.2 \mathrm{c}, \mathrm{C}, \mathrm{g}$ & $2.4 \pm 0.1 \mathrm{a}, \mathrm{B}, \mathrm{h}$ & $0.73 \pm 0.01 \mathrm{a}, \mathrm{A}, \mathrm{h}$ & $1.22 \pm 0.11 b, A, g$ & $428.9 \pm 46.4$ b,B,g & $343.3 \pm 28.4$ b,A,h \\
\hline & 3 & $2.8 \pm 0.2 b, \mathrm{~B}, \mathrm{~g}$ & $2.2 \pm 0.2 b, \mathrm{~B}, \mathrm{~h}$ & $0.85 \pm 0.01 d, \mathrm{C}, \mathrm{h}$ & $1.60 \pm 0.09 d, \mathrm{~B}, \mathrm{~g}$ & $271.6 \pm 100.5 a b, A, g$ & $181.0 \pm 20.0 a, B, h$ \\
\hline
\end{tabular}

*mean value \pm standard deviation

†H.D.:harvest date

$a, b, c, d, e:$ Mean values of the same harvest date and the same crop year with a different superscript differ significantly $(P \leq 0.05)$ [comparison between varieties]

$\mathrm{A}, \mathrm{B}, \mathrm{C}$ : Mean values of the same variety and the same crop year with a different superscript differ significantly $(\mathrm{P} \leq 0.05)[\mathrm{comparison}$ between harvest dates]

$\mathrm{g}, \mathrm{h}$ : Mean values of the same variety and the same harvest date with a different superscript differ significantly $(\mathrm{P} \leq 0.05)$ [comparison between crop years]

The normal, bold and italic small letters refer to the first, second and third harvest dates, respectively.

the following crop year, and the olives were found to have lower contents of the remaining four compounds in 2007 than they contained in 2006. All the phenolic compounds determined in this assay had higher values in 2007 than in 2006 for the olives cultivated in Alanya. However, the phenolic compounds in the other three location samples generally decreased in the following crop season when compared to the previous season.

\subsection{Antioxidant capacity, radical scavenging effect and total phenolics}

The Trolox equivalent antioxidant capacity, DPPH radical scavenging effect and total phenolic contents of the fruits of the Sarıulak variety from four different locations are shown in Table 2. In general Silifke and Karaman fruits showed the highest antioxidant capacity, whereas the fruits derived from Ceyhan showed the lowest antioxidant capacity values in the first and second HDs in both the 2006 and 2007 crop years.

The total phenolics of the fruits were determined within $180.5-476.0 \mathrm{mg}$ gallic acid/ $\mathrm{kg}$ fresh fruit. Karaman and Silifke fruits had the highest total phenolic values on the second and third HDs in 2006. The highest total phenolic content was determined in the Alanya samples on the second $\mathrm{HD}$ in 2007, as the values were not significant for the first and third HDs in 2007. Cerretani et al. (2004) reported a decrease from 441.4 to $209.5 \mathrm{mg} /$ $\mathrm{kg}$ and 585.2 to $409.8 \mathrm{mg} / \mathrm{kg}$ in the total phenolics of the Nostrana di Brisighella and Ghiacciolo varieties from Italy harvested between October and December. The total phenolic values determined for the fruits of the Sarıulak variety in this study were closer to those reported by Cerretani et al. (2004) and were lower than those of the Italian varieties concerning some HDs and crop years.

The influence of location on the free-radical scavenging effect was different in relation to the crop year. Ceyhan fruits showed the lowest DPPH radical scavenging ability in 2006 , while the lowest $\mathrm{DPPH}$ radical scavenging abilities were detected for the fruits from Karaman and Silifke in 2007 in all the three HDs. In general Silifke and Karaman fruits showed higher antioxidant activity and free-radical scavenging effect than those of the fruits of other locations. These locations contained higher levels of oleuropein, vanillic and cinnamic acids, which might have a role in the higher antioxidant activity of these fruits. Keçeli and Gordon (2001) compared the DPPH radical scavenging effect of Sarlulak fruits from the Aegean and Mediterranean regions of Turkey and concluded that the extract from the Aegean fruits was slightly more effective than the extract of the Mediterranean fruits. The significant effect of the plantation place on parameters such as phenolic content and antioxidant activity of olive fruits was also reported by Baiano et al. (2009).

When comparing the results regarding the HD, there were not significant differences in total phenolics 
of the fruits, while there were slight decreases for the Silifke samples and increases for the Ceyhan samples towards the later HDs in 2007. A reduction was determined in the free-radical scavenging effect of fruits harvested in the Alanya and Karaman locations when the harvest time progressed; while there was a raise for the Ceyhan fruits when the HD moved on. The free-radical scavenging effect of the fruits from the Silifke location was low at the beginning, followed by an increase on the second $\mathrm{HD}$ and then it decreased until the end of last HD. All of the locations showed similar changes in antioxidant activity pointing to an increase through the later HDs.

Generally, the differences in total phenol content, radical scavenging effect and antioxidant activity of fruits from different locations between the two consecutive crop years were statistically significant. The Alanya and Ceyhan samples showed higher while Silifke samples showed lower radical scavenging effects and total phenolic contents in the following crop year. The lower average rainfall in the consecutive crop year in Silifke could bring about the drop in total phenolic content of fruits from this location due to the positive relationship between phenol content and precipitation which was previously reported by Ben Temime et al. (2006). Because Silifke was the only location which had a decrease in the rainfall level between crop years a reduction in total phenolic content was seen only in Silifke samples. There was not a significant difference in total phenolic content of the Karaman samples in terms of crop year. Antioxidant activity of the samples decreased in the following crop year, except for Alanya samples which showed higher antioxidant activity in 2007 than the previous year. This might be connected to the individual phenolic content of Alanya fruits, as the concentrations of all the phenolic compounds in fruits from this location increased in the following crop year, unlike the other location samples.

\section{CONCLUSIONS}

As compared to the fruits from the Ceyhan, Silifke and Karaman locations, the fruits from the Alanya location produced higher levels of individual phenolic compounds, while the fruits from the Ceyhan locations revealed lower levels of these compounds. The fruits obtained from trees cultivated in Ceyhan showed the lowest antioxidant activity, radical scavenging effect and total phenolics content.

The effect of HD on phenolic compounds such as 4-hydroxyphenyl acetic acid, caffeic acid, syringic acid and h-tyrosol, luteolin, chlorogenic acid and 3,4-dihydroxybenzoic acid identified in this work appeared to be more dependent on the location factor. The antioxidant activity of fruits increased with progressing HD. Total phenolics in olives generally showed no significant differences the HDs.

The changes in verbascoside and rutin according to crop year were in an increasing trend in all the samples in the following crop year, while luteolin, cinnamic acid, 4-hydroxyphenyl acetic acid and 4-hydroxybiphenyl carboxylic acid contents of the olives were found to be lower in 2007 than in 2006. All the phenolic compounds determined in this assay had higher values in 2007 than in 2006 for the olives cultivated in Alanya. However, the phenolic compounds in the other three location samples generally decreased in the following crop year when compared to the previous year. The olive fruits from Alanya had higher antioxidant activity, radical scavenging effect and total phenolics in the following crop year which could be attributed to the same trend determined for all the individual phenolics of fruits obtained from this location.

\section{ACKNOWLEDGEMENTS}

This study is a part of the PhD thesis of Derya Arslan and was financially supported by Selçuk University Office of Scientific Research Projects (Project ID: SÜ-BAP-07101016).

\section{REFERENCES}

Amiot MJ, Fleuriet A, Macheix JJ. 1986. Importance and evolution of phenolic compounds in olive during growth and maturation. J. Agric. Food Chem. 34, 823-826.

Anonymous 1991. Standart Zeytin Çeşitleri Katalogu. Tarım ve Köyişleri Bakanlığı Yayınları. No: 334, Seri: 16. Ankara.

Artajo LS, Romero MP, Motilva MJ. 2006. Transfer of phenolic compounds during olive oil extraction in relation to ripening stage of the fruit. J. Sci. Food Agric. 86, 518-527.

Baiano A, Gambacorta G, Terracone C, Previtali MA, La Notte E. 2009. Characteristics of drupes, phenolic content and antioxidant capacity of Italian olive fruits. J. Food Lipids. 16, 209-226.

Ben Temime S, Taamalli W, Baccouri B, Abaza L, Daoud D, Zarrouk M. 2006. Changes in olive oil quality of Chétoui variety according to origin of plantation. $J$. Food Lipids. 13, 88-99.

Boskou D. 1996. The olive oil composition. In: Olive Oil, Pp. 5-18. Champaign, IL: AOCS Press.

Boskou D. 2006. Olive oil chemistry and technology. AOCS Press, Champaign, IL, USA.

Botía JM, Ortuño A, Benavente-García O, Báidez AG, Frías J, Marcos D, Del Río JA. 2001. Modulation of the biosynthesis of some phenolic compounds in Olea europaea L. fruits: Their influence on olive oil quality. J. Agric. Food Chem. 49, 355-358.

Cerretani L, Bendini A, Rotondi A, Mari M, Lercker G, Toschi T. 2004. Evaluation of the oxidative stability and organoleptic properties of extra-virgin olive oils in relation to olive ripening degree. Progr. Nutr. 6, 5056.

DiE 2008. Devlet İstatistik Enstitüsü (Turkish Statistical Institute) Crop Production Statistics, Ankara, Türkiye (http://www.turkstat.gov.tr/PreTablo.do?tb_id=45\&ust_ id $=13$ )

Esti M, Cinquanta L, La Notte E. 1998. Phenolic compounds in different olive varieties. J. Agric. Food Chem. 46, 32-35. 
Gomez-Rico A, Fregapane G, Salvador MD. 2008. Effect of cultivar and ripening on minor components in Spanish olive fruits and their corresponding virgin olive oils. Food Res. Int. 41, 433-440.

Gurbuz S, Kiran-Ciliz N, Yenigun O. 2004. Cleaner production implementation through process modifications for selected SMEs in Turkish olive oil production. J. Clean Prod. 12, 613-621.

Keçeli T, Gordon MH. 2001. The antioxidant activity and stabilility of the phenolic fraction of green olives and extra virgin olive oil. J. Sci. Food Agric. 81, 13911396.

Loumou A, Giourga C. 2003. Olive groves: "The life and identity of the Mediterranean". Agr. Hum. Val. 20, 87-95.

Miller N, Rice-Evans C. 1997. Factors influencing the antioxidant activity determined by the ABTS $\bullet+$ radical cation assay. Free Radic. Res. 26, 195-199.

Mousa MY, Gerosopoulos D, Metzidakis I, Kiritsakis A. 1996. Effect of altitude on fruit and quality characteristics of "Mastoides" olives. J. Sci. Food Agric. 71, 345-350.

Nergiz C, Engez Y. 2000. Compositional variation of olive fruit during ripening. Food Chem. 69, 55-59.

Ocakoğlu D, Tokatı F, Özen B, Korel F. 2009. Distribution of simple phenols, phenolic acids and flavonoids in Turkish monovarietal extra virgin olive oils for two harvest years. Food Chem. 113, 410-416.

Öğütçü M, Mendes M, Yılmaz E. 2008. Sensorial and physico-chemical characterization of virgin olive Oils Produced in Çanakkale. J. Am. Oil Chem. Soc. 85, 441-456.

Özkaya MT. 2003. Standart zeytin çeşitlerimiz ve bazı özellikleri. (Editör S. Batal) Zeytin yetiştiriciliği, Hasad Yayıncılık, Ankara.

Ryan D, Robards K. 1998. Phenolic Compounds in Olives. Analyst. 123, 31-44

Ryan D, Robards K, Lavee S. 1999. Changes in phenolic content of olive during maturation. International $J$. Food Sci. Technol. 34, 265-274.

Ryan D, Antolovich M, Prenzler P, Robards K, Lavee S. 2002. Biotransformations of phenolic compounds in Olea europaea L. Sci. Hort. 92, 147-176.

Romani A, Mulinacci N, Pinelli P, Vincieri F, Cimato A. 1999. Polyphenolic content in five Tuscany cultivars of Olea europaea L. J. Agric. Food Chem. 47, 964-967.

Romero C, García P, Brenes M, García A, Garrido A. 2002. Phenolic compounds in natural black Spanish olive varieties. Eur. Food Res. Technol. 215, 489-496.

Romero PM, Jesus Tovar M, Ramo T, Jose Moltiva M. 2003. Effect of crop season on the composition of virgin olive oil with protected designation of origin "Les Garrigues". J. Am. Oil Chem. Soc. 80, 423-430.

Servili M, Baldioli M, Selvaggini R, Miniati E, Macchioni A, Montedoro G. 1999. High-performance liquid chromatography evaluation of phenols in olive fruit, virgin olive oil, vegetation waters, and pomace and $1 \mathrm{D}$ and 2D nuclear magnetic resonance characterization. J. Am. Oil Chem. Soc. 76, 873-882.

Servili M, Selvaggini R, Esposto S, Taticchi A, Montedoro G, Morozzi G. 2004. Health and sensory properties of virgin olive oil hydrophilic phenols: Agronomic and technological aspects of production that affect their occurrence in the oil. J. Chrom. A. 1054, 113-127.

Silva S, Gomes L, Leitão F, Coelho AV, Boas LV. 2006. Phenolic compounds and antioxidant activity of Olea europea L. fruits and leaves. Food Sci. Technol. Int. 12, 385-396.

Singh RP, Murthy KNC, Jayaprakasha GK. 2002. Studies on the antioxidant activity of pomegranate (Punica granatum) peel and seed extracts using invitro models. J. Agric. Food Chem. 50, 81-86.

Soler-Rivas C, Espín JC, Wichers HJ. 2000. Oleuropein and related compounds. J. Sci. Food Agric. 80, 10131023.

Tanılgan K, Özcan MM, Ünver A. 2007. Physical and chemical characteristics of five Turkish olive (Olea europea L.) varieties and their oils. Grasas Aceites. 5, $142-147$.

Tetik HD. 1989. Table Olive Technology in Turkey. Olivea. 27, 23-24.

Tovar MJ, Romero MP, Alegre S, Girona J, Motilva MJ. 2002. Composition and organoleptic characteristics of oil from Arbequina olive (Olea europaea L.) trees under deficit irrigation. J. Sci. Food Agric. 82, 17551763.

Ünal K, Nergiz C. 2003. The effect of table olive preparing methods and storage on the composition and nutritive value of olives. Grasas Aceites. 54, 71-76.

Vasquez Roncero A, Janer Del Valle C, Janer Del Valle ML. 1973. Determinacion de los polifenoles totales del aceite de oliva. Grasas Aceites. 24, 350-357.

Vinha AF, Silva BM, Andrade PB, Seabra RM, Pereira JA, Oliveira MB. 2002. Development and evaluation of an HPLC/DAD method for the analysis of phenolic compounds from olive fruits. J. Liq. Chrom. Relat. Tech. 25, 151-160.

Vinha AF, Ferreres F, Silva BM, Valentao P, Gonçalves A, Pereira JA, Oliveria MB, Seabre RM, Andrade PB. 2005. Phenolic profiles of Portuguese olive fruits (Olea europea L.): Influence of cultivar and geographical origin. Food Chem. 89, 561-568.

Yousfi K, Cert RM, Garcìa JM. 2006. Changes in quality and phenolic compounds of virgin olive oils during objectively described fruit maturation. Eur. Food Res. Technol. 223, 117-124.

ZAE Olive Research Institute of Turkey 2007. Economics and Statistics of Olive Oil in the World and Turkey. http://www.zae.gov.tr. Accessed Jan 2010.

Recibido: $24 / 3 / 11$ Aceptado: $19 / 5 / 11$ 